Draft VERSION FEBRUARY 5, 2020

Typeset using LATEX preprint2 style in AASTeX61

\title{
DISCOVERY OF A GIANT RADIO FOSSIL IN THE OPHIUCHUS GALAXY CLUSTER
}

\author{
S. Giacintucci, ${ }^{1}$ M. Markevitch, ${ }^{2}$ M. Johnston-Hollitt,${ }^{3,4}$ D. R. Wik, ${ }^{5}$ Q. H. S. Wang, ${ }^{5}$ And \\ T. E. Clarke ${ }^{1}$ \\ ${ }^{1}$ Naval Research Laboratory, 4555 Overlook Avenue SW, Code 7213, Washington, DC 20375, USA \\ ${ }^{2}$ NASA/Goddard Space Flight Center, Greenbelt, MD 20771, USA \\ ${ }^{3}$ International Centre for Radio Astronomy Research, Curtin University, Bentley, WA 6102, Australia \\ ${ }^{4}$ Peripety Scientific Ltd., PO Box 11355 Manners Street, Wellington 6142, New Zealand \\ ${ }^{5}$ Department of Physics and Astronomy, University of Utah, 115 South 1400 East, Salt Lake City, UT 84112, USA
}

(Received November 19, 2019; Revised January 8, 2020; Accepted February 5, 2020)

Submitted to ApJ

\begin{abstract}
The Ophiuchus galaxy cluster exhibits a curious concave gas density discontinuity at the edge of its cool core. It was discovered in the Chandra X-ray image by Werner and collaborators, who considered a possibility of it being a boundary of an AGN-inflated bubble located outside the core, but discounted this possibility because it required much too powerful an AGN outburst. Using low-frequency (72-240 MHz) radio data from MWA/GLEAM and GMRT, we found that the Xray structure is, in fact, a giant cavity in the X-ray gas filled with diffuse radio emission with an extraordinarily steep radio spectrum. It thus appears to be a very aged fossil of the most powerful AGN outburst seen in any galaxy cluster $\left(p V \sim 5 \times 10^{61}\right.$ erg for this cavity). There is no apparent diametrically opposite counterpart either in X-ray or in the radio. It may have aged out of the observable radio band because of the cluster asymmetry. At present, the central AGN exhibits only a weak radio source, so it should have been much more powerful in the past to have produced such a bubble. The AGN is currently starved of accreting cool gas because the gas density peak is displaced by core sloshing. The sloshing itself could have been set off by this extraordinary explosion if it had occurred in an asymmetric gas core. This dinosaur may be an early example of a new class of sources to be uncovered by low-frequency surveys of galaxy clusters.
\end{abstract}

Keywords: catalogs — galaxies: clusters: general — surveys — X-rays: galaxies: clusters - radio continuum: galaxies: clusters

Corresponding author: Simona Giacintucci

simona.giacintucci@nrl.navy.mil 


\section{INTRODUCTION}

The hot and massive Ophiuchus cluster of galaxies, the second X-ray brightest cluster in the sky, has a cool core with a very steep temperature drop from $10 \mathrm{keV}$ to below $1 \mathrm{keV}$ (e.g., Pérez-Torres et al. 2009, hereafter P09; Werner et al. 2016, hereafter W16). A high-resolution $\mathrm{X}$-ray image of the Ophiuchus core region, with interesting features marked, is shown in Fig. 1, derived using the same data as in W16. The core exhibits several prominent sloshing X-ray cold fronts - concentric gas density edges at various radii (Ascasibar \& Markevitch 2006; ZuHone et al. 2010; Million et al. 2010; W16). The core also exhibits a radio synchrotron minihalo (Govoni et al. 2009, Murgia et al. 2010, hereafter G09 and M10), similarly to most cool cores in massive clusters (Giacintucci et al. 2017). The same population of ultra-relativistic electrons may explain the reported nonthermal hard X-ray emission (Eckert et al. 2008; Nevalainen et al. 2009; Fujita et al. 2008). The Chandra high-resolution X-ray image revealed that sloshing has displaced the peak of the cluster cool gas away from the $\mathrm{cD}$ galaxy (ZuHone et al. 2010; Million et al. 2010), apparently depriving the currently active galactic nucleus (AGN) of its accretion fuel, which is probably why the AGN is currently weak both in the radio and in the X-ray (Hamer et al. 2012; W16).

Another curious feature noted by W16 in their deep Chandra X-ray image of the Ophiuchus center is a concave gas density edge at the boundary of the cool core (Fig. 1). W16 briefly considered a possibility that this is a bubble blown by jets from the central AGN, similar to Perseus (Fabian et al. 2003), Hydra A (Nulsen et al. 2005), MS 0735+74 (McNamara et al. 2005) and numerous other examples found in clusters and massive elliptical galaxies (e.g., Rafferty et al. 2006). Such $\mathrm{X}$-ray cavities are usually filled with extended

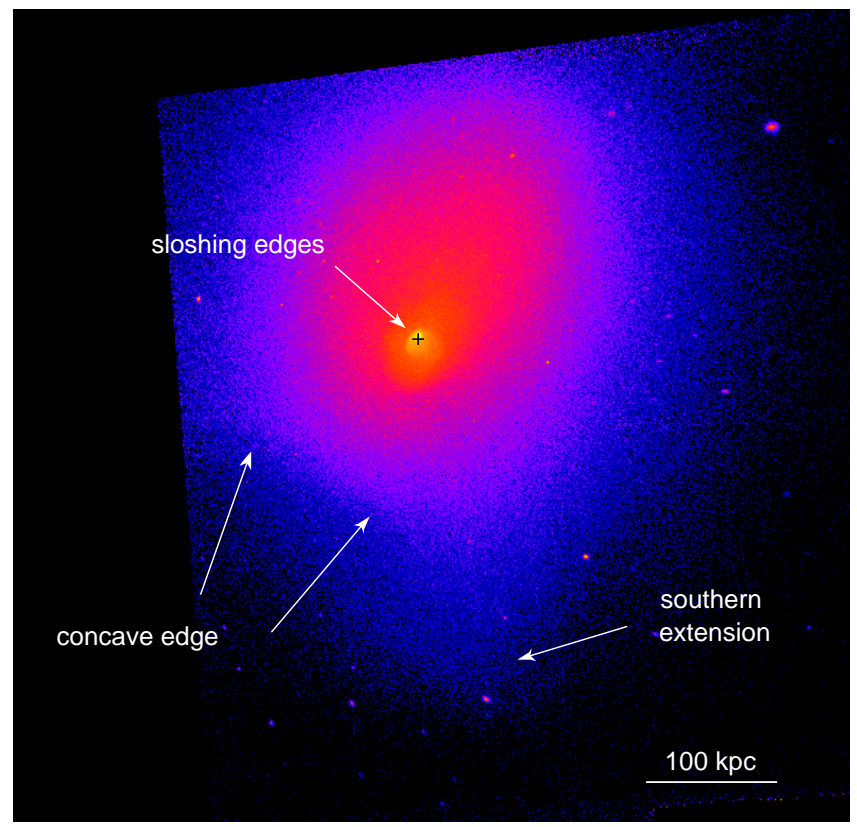

Figure 1. Chandra X-ray image of the Ophiuchus central region in the $0.5-4 \mathrm{keV}$ band, with main features marked. Black cross marks the $\mathrm{cD}$ galaxy; the $\mathrm{X}$-ray peak is displaced from that position, apparently by vigorous gas sloshing, as evidenced by several prominent concentric brightness edges at different radii. The subtle concave edge, noticed by $\mathrm{W} 16$, is the subject of this work.

radio synchrotron emission from the ultrarelativistic electrons ejected by the AGN. The radio spectrum of this emission steepens as these electrons cool on 10-100 Myr timescales (e.g., Murgia et al. 2011). M10 did find a hint of such extended radio emission near the edge of the putative cavity (their "source E"), but interpreted it as a steep-spectrum outlying region of the central radio minihalo. W16 estimated the diameter of the cavity from the curvature of the X-ray edge and the $p V$ work $^{1}$ required to displace the intracluster medium (ICM) to create such a cavity, $5 \times 10^{61} \mathrm{erg}$, which turns out to be several times greater than that for the most powerful unambiguous AGN outburst known, MS 0735+74 (McNamara et al. 2005).

\footnotetext{
${ }^{1} p$ is the pressure of the gas surrounding the cavity and $V$ is the cavity's volume.
} 
They argued that such an explosion would very likely have wiped out the cool core, which still exists and exhibits sharp gradients of entropy and metallicity. Therefore, they concluded that the feature is more plausibly a hydrodynamic effect of a cluster merger, not unlike the KelvinHelmholtz eddy at the edge of the Perseus core (Walker et al. 2017), which are occasionally seen in hydro simulations of cluster core sloshing (e.g., Ascasibar \& Markevitch 2006).

In this paper, we inspect low-frequency radio images from the Murchison Widefield Array (MWA), Giant Metrewave Radio Telescope (GMRT) and Very Large Array (VLA) — where the aged relativistic particles from past AGN activity might still leave trace emission - compare them with the X-ray images, and uncover a fossil of an extraordinarily powerful AGN outburst.

We use a $\Lambda$ CDM cosmology with $\mathrm{H}_{0}=70 \mathrm{~km}$ $\mathrm{s}^{-1} \mathrm{Mpc}^{-1}, \Omega_{m}=0.3$ and $\Omega_{\Lambda}=0.7$. At the redshift of Ophiuchus $(z=0.028), 1^{\prime \prime}=0.562$ kpc. All errors are quoted at the $68 \%$ confidence level. The radio spectral index $\alpha$ is defined according to $S_{\nu} \propto \nu^{-\alpha}$, where $S_{\nu}$ is the flux density at the frequency $\nu$.

\section{RADIO DATA}

Intrigued by the W16 estimate of the enormous energy output required to displace the cluster X-ray gas if the concave X-ray edge were a cavity, we have searched the low-frequency radio data for faint diffuse signal from aged ultrarelativistic particles ejected by this hypothetical AGN explosion. Indeed, the low-frequency images from the MWA GLEAM survey ${ }^{2}$ in several bands covering $72-230 \mathrm{MHz}$ reveal a large, extended radio source cospatial with the putative X-ray cavity. This source was not detected in previously published radio images (P09, G09, $\mathrm{M} 10$ ) and extends far beyond the source $\mathrm{E}$ in

2 MWA GaLactic and Extragalactic All-sky MWA (Wayth et al. 2015, Hurley-Walker et al. 2017).
M10. This prompted us to reanalyze and combine the archival GMRT data used in P09 and M10 in order to reach higher sensitivity and investigate the nature of this emission and its connection to the X-ray cavity. Below we compare our rederived, lower-noise GMRT images at 153 $\mathrm{MHz}$ and $240 \mathrm{MHz}$ to the GLEAM images, as well as to a $74 \mathrm{MHz}$ image from the VLSSr survey. ${ }^{3}$ We also use an image at $1477 \mathrm{MHz}$ from the archival VLA D-configuration observation that was rederived in Giacintucci et al. (2019, hereafter G19) from the data used in G09 and M10.

The observations used in our analysis provide sensitivity to angular structures as large as $36^{\prime}$ (VLSSr) or larger $\left(44^{\prime}-68^{\prime}\right.$ for the GMRT and several degrees for GLEAM), which correspond to a physical scale of at least $1.2 \mathrm{Mpc}$ at the redshift of Ophiuchus. The largest linear scale detectable by the VLA D-configuration observation at $1477 \mathrm{MHz}$ is significantly smaller $(\sim 500$ $\mathrm{kpc})$.

\subsection{GMRT}

We obtained from the GMRT archive the observations of the Ophiuchus cluster at $153 \mathrm{MHz}$ and $240 \mathrm{MHz}$ used by M10 and P09 and reduced them using the $\mathrm{NRAO}^{4}$ Astronomical Image Processing System (AIPS). Details on these observations are summarized in Table 1.

The data were collected in spectral-line observing mode, using the GMRT hardware backend, with a total observing bandwidth of 8 $\mathrm{MHz}$. We carefully inspected the data and found that all observations were partially impacted by radio frequency interference (RFI). We used RFLAG to excise RFI-affected visibilities, followed by manual flagging to remove residual bad data. Gain and bandpass calibrations were applied using the standard primary

\footnotetext{
3 VLA Low-Frequency Sky Survey Redux (Lane et al. 2014).

${ }^{4}$ National Radio Astronomy Observatory.
} 
calibrators $3 \mathrm{C} 286$ at $153 \mathrm{MHz}$ and $3 \mathrm{C} 286$ and 3C48 at $240 \mathrm{MHz}$. The Scaife \& Heald (2012) scale was adopted in SETJY to set their flux densities at these frequencies. Phase calibrators, observed several times during the observation, were used to calibrate the data in phase. To reduce the size of the data sets, minimizing at the same time the effects of bandwidth smearing, the calibrated visibilities were averaged appropriately in frequency, with 14 channels at $240 \mathrm{MHz}$ and 21 channels at $153 \mathrm{MHz}$, each $0.375 \mathrm{MHz}$ and $0.25 \mathrm{MHz}$ wide, respectively. A number of phase self-calibration iterations, followed by a final self-calibration step in amplitude and phase, were applied to the target visibilities. During the self-calibration process, images were made using wide-field imaging, decomposing the primary beam area into a large number of smaller facets. Additional facets were placed on outlier bright sources out to a distance of 10 degrees from the phase center. The final images were produced using widefield and multi-resolution imaging in AIPS with the Briggs robust weighting (Briggs 1995) set to 0 . At $240 \mathrm{MHz}$, we combined the two final self-calibrated data sets in the $u v$ plane to produce the final images. The root mean square $(r m s)$ noise levels reached in our full-resolution images are reported in Table 1. Our values are significantly lower $(\sim 40 \%-50 \%)$ than the noise levels reached by M10 and P09. Finally, correction for the GMRT primary beam response was applied using PBCOR in AIPS $^{5}$.

Residual amplitude errors, $\sigma_{\text {amp }}$, are estimated to be within $15 \%$ at $153 \mathrm{MHz}$ and $10 \%$ at $240 \mathrm{MHz}$ (Chandra, Ray \& Bhatnagar 2004). Errors on measured flux densities were calculated as

$$
\sigma_{S_{\nu}}=\sqrt{\left(\sigma_{\mathrm{amp}} \times S_{\nu}\right)^{2}+\left(\sigma_{r m s} \times \sqrt{N_{\text {beam }}}\right)^{2}},
$$

\footnotetext{
${ }^{5}$ http://www.ncra.tifr.res.in:8081/ ${ }^{\sim}$ ngk/ primarybeam/beam.html.
}

where $S_{\nu}$ is the source flux density at the frequency $\nu, \sigma_{r m s}$ is the noise level in the image, and $N_{\text {beam }}$ is the number of beams crossing the source.

\subsection{GMRT, MWA and VLA images}

Figure 2 presents our GMRT images at 240 $\mathrm{MHz}$ at a resolution of $45^{\prime \prime}(a)$ and $120^{\prime \prime}(b)$. The radio emission is dominated by three bright extended radio galaxies. A detailed morphological and spectral study of these sources has been presented by M10. The central diffuse minihalo (G09, M10) is well detected in our images, where it occupies an area of $\sim 200$ $\mathrm{kpc}$ in radius. The location of the central $\mathrm{cD}$ galaxy (yellow cross) coincides with the faint radio point source embedded in the minihalo emission (M10, W16).

A distinct region of extended emission (hereafter referred to as relic lobe) is visible SouthEast of the cluster center. Its brightest part (ridge) coincides with source E in M10, but we see a considerably larger source than in M10, thanks to the higher sensitivity of our GMRT images. The relic lobe extends out to a distance $d \sim 17^{\prime}(\sim 570 \mathrm{kpc})$ from the cluster center and has a radius of $r \sim 6 .^{\prime} 8(\sim 230 \mathrm{kpc})$. A GMRT image at $153 \mathrm{MHz}$ at the resolution of $120^{\prime \prime}$ is shown in Figure 3. Due to the lower sensitivity of this image, only the innermost part of the minihalo $(r \sim 130 \mathrm{kpc})$ and the brightest portion of the relic lobe $(d \sim 400 \mathrm{kpc})$ are well detected.

In Figure 4, we overlay our GMRT $240 \mathrm{MHz}$ contours on the low-frequency continuum images from GLEAM in the $72-103 \mathrm{MHz}, 103-$ $134 \mathrm{MHz}, 139-170 \mathrm{MHz}$ and $170-231 \mathrm{MHz}$ subbands. At the lowest GLEAM frequencies (panels $a$ and $b$ ), the relic lobe is fully detected with good spatial coincidence with the GMRT image. The 139-170 MHz and 170-231 MHz sub-band images, instead, detect well only its northern ridge. The ridge is also visible in the VLSSr image at $74 \mathrm{MHz}$, shown in Fig. 5. Its peak 
Table 1. Newly analyzed GMRT observations

\begin{tabular}{cccccccc}
\hline \hline $\begin{array}{c}\text { Project } \\
\text { code }\end{array}$ & $\begin{array}{c}\text { Frequency } \\
(\mathrm{MHz})\end{array}$ & $\begin{array}{c}\text { Bandwidth } \\
(\mathrm{MHz})\end{array}$ & Observation & Time & $\begin{array}{c}\text { FWHM } \\
(\text { hour })\end{array}$ & $\begin{array}{c}\left(^{\prime \prime} \times^{\prime \prime}\right) \\
(\mathrm{mJy})\end{array}$ & $\begin{array}{c}\Theta_{\text {LAS }} \\
\left({ }^{\prime}\right)\end{array}$ \\
\hline 14MPA03 & 153 & 8 & 2008 Aug 21 & 3.8 & $27 \times 22$ & 3.0 & 68 \\
RAS + 14MPA03 & 240 & 8 & 2008 May 24, Aug 23,28 & 11.9 & $15 \times 12$ & 0.55 & 44 \\
\hline
\end{tabular}

Note-Column 1: project code. Columns 2 and 3: Observing central frequency and bandwidth. Column 4: Observation dates. Column 5: Total time on source. Columns 6 and 7: Full width half maximum (FWHM) of the radio beam and $r m s$ level $(1 \sigma)$ of the final images at full array resolution. Column 8: largest angular scale detectable by the array. The cluster center coincides with the radio point source embedded in the diffuse minihalo and associated with the central $\mathrm{cD}$ galaxy (yellow cross in Fig. 2a).
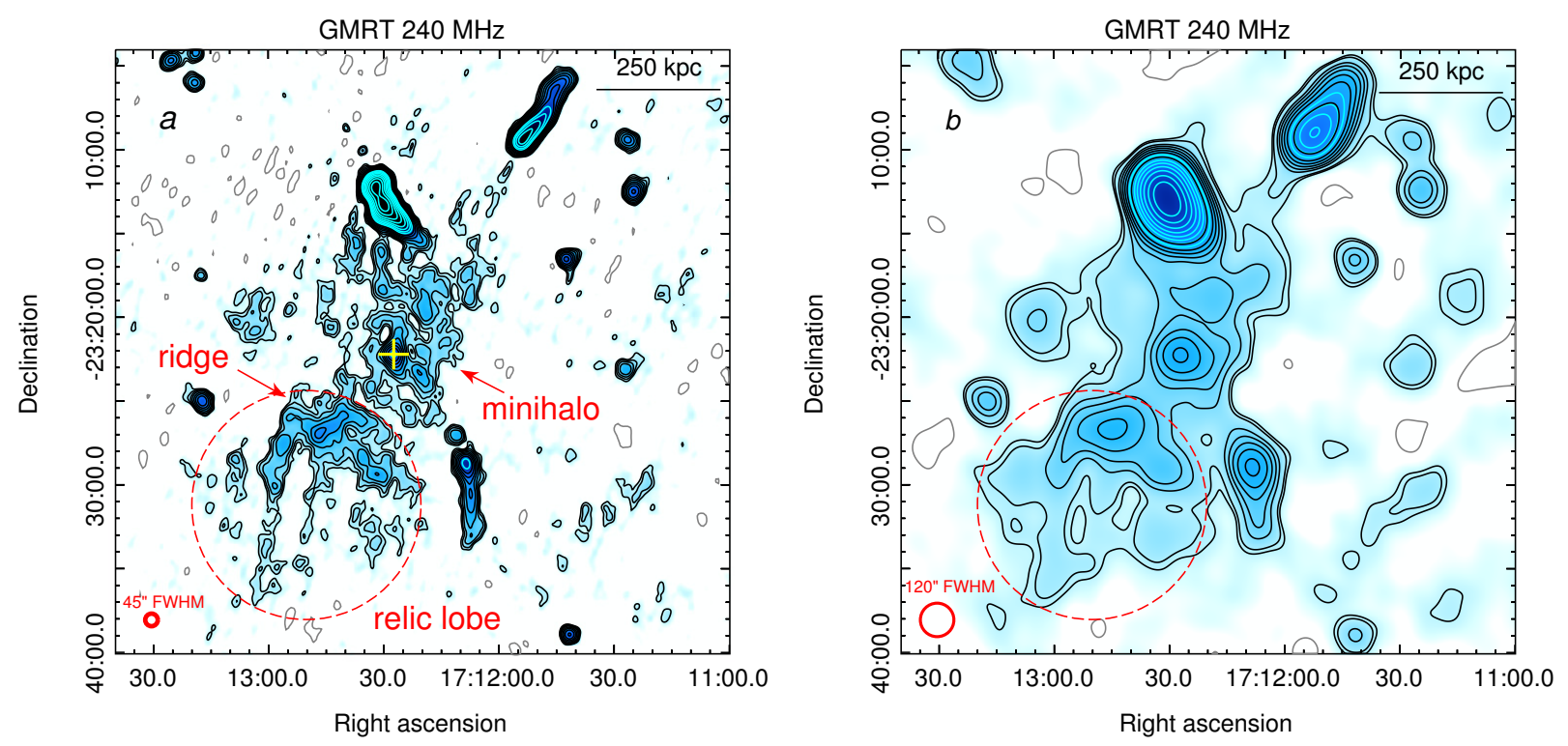

Figure 2. GMRT radio images at $240 \mathrm{MHz}$ at the resolution of $45^{\prime \prime}(a)$ and $120^{\prime \prime}(b)$. The restoring beam is also shown in red in the bottom-left corner of each image. The $r m s$ noise $1 \sigma$ level is 1.3 beam $^{-1}$ and $3.3 \mathrm{mJy}_{\text {beam }}{ }^{-1}$, respectively. Contours (black and cyan) are spaced by a factor of $\sqrt{2}$, starting from $+3 \sigma$. Contours at $-3 \sigma$ are shown as grey. The radius of the dashed, red circle is $6^{\prime \prime} .8=230 \mathrm{kpc}$. The yellow cross in panel $a$ marks the position of the central cD galaxy.

emission is detected at a $4 \sigma$ level $(1 \sigma=180 \mathrm{mJy}$ beam $\left.^{-1}\right)$. The fainter emission from the minihalo is not detected at this sensitivity level. Finally, in Figure 6 we compare the $240 \mathrm{MHz}$ emission to a higher-frequency VLA image at 1477 $\mathrm{MHz}$ (from G19), showing the central minihalo and only hints of the much lower surface brightness emission from the relic lobe.

\section{RADIO ANALYSIS}

\subsection{Flux density of the relic lobe}

We measured the flux density of the relic lobe in the GMRT, GLEAM, VLSSr and 


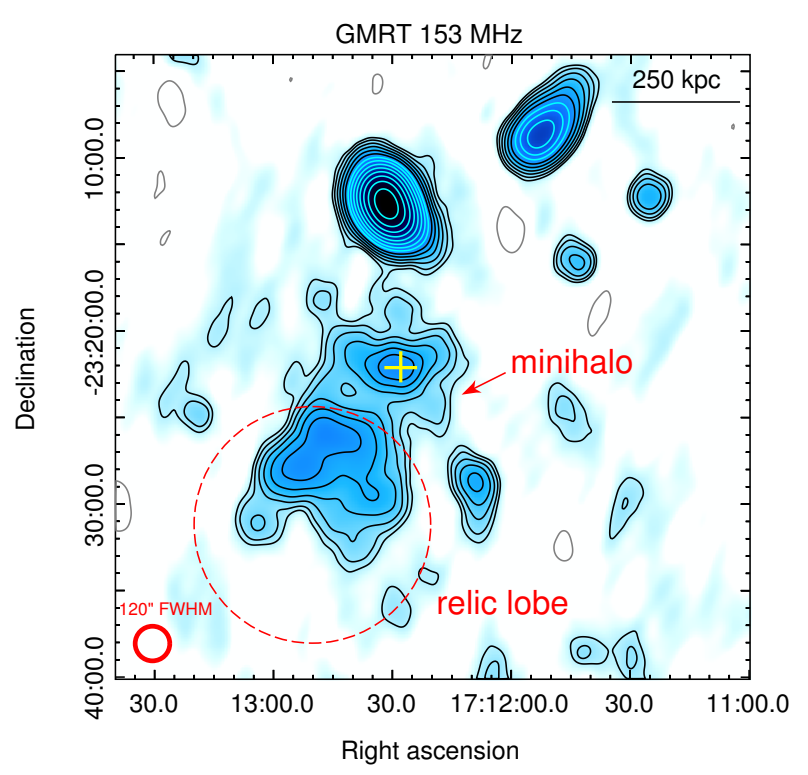

Figure 3. GMRT radio image at $153 \mathrm{MHz}$ at $120^{\prime \prime}$ resolution. The restoring beam is also shown in red in the bottom-left corner. The rms noise $1 \sigma$ level is $12 \mathrm{mJy}$ beam $^{-1}$. Contours are spaced by a factor of $\sqrt{2}$, starting from $+3 \sigma$. Contours at $-3 \sigma$ are shown as grey. The radius of the dashed, red circle is $6^{\prime \prime} .8=230 \mathrm{kpc}$ (as in Fig. 2). The yellow cross marks the position of the central $\mathrm{cD}$ galaxy.

VLA images within a circular region of radius $r=6^{\prime} .8$, centered on $\mathrm{RA}_{\mathrm{J} 2000}=17 \mathrm{~h} 12 \mathrm{~m} 50.2 \mathrm{~s}$ and $\mathrm{DEC}_{\mathrm{J} 2000}=-23 \mathrm{~d} 31 \mathrm{~m} 13 \mathrm{~s}$. This region corresponds to the X-ray cavity size inferred from the $X M M-N e w t o n$ image (Fig. 10) and is entirely filled by the relic lobe at $240 \mathrm{MHz}$ (Fig. 2). Table 2 summarizes the relic lobe flux density at all frequencies. Errors include the local image rms and flux calibration uncertainty (Eq. 2.1). Differences between the flux-density scales adopted for the GMRT and VLSSr (Scaife \& Heald 2012), GLEAM (Baars et al. 1977) and VLA data (Perley \& Butler 2014) are estimated to be at most $\sim 5 \%$ (Perley \& Butler 2017).

For the VLSSr, we corrected the flux measured on the image for the clean bias and adopted a flux uncertainty of $20 \%$, as appropriate for extended sources detected at a very low signal-to-noise ratio (Lane et al. 2014). This high uncertainty also accounts for the source location at the edge of the VLSSr mosaic, as fluxes can be affected by uncertainties in the primary-beam model at large distances from the pointing center.

For GLEAM, a systematic flux uncertainty of $8 \%$ is expected for sources in the same declination range as the Ophiuchus cluster (HurleyWalker et al. 2017). Due to the very low Galactic latitude of Ophiuchus, the GLEAM fluxes reported in Table 2 have been corrected for contamination due to Galactic diffuse synchrotron emission, to which GLEAM is highly sensitive thanks to the very short $u v$ spacings of the MWA (e.g., Su et al. 2017). The GLEAM images of the region containing the Ophichus cluster exhibit in fact large-scale structures of Galactic emission, which affect flux density measurements. Fig. 7 shows a $3^{\circ} \times 3^{\circ}$ region, centered on Ophiuchus, of the $170-231 \mathrm{MHz}$ GLEAM image, where large variations of foreground emission are visible across the image. In particular, the Ophiuchus cluster appears to be located at the boundary of a bright region of Galactic emission. To estimate the contribution of this Galactic foreground, we have extracted the flux density within a set of 8 circular source-free regions of the same size as the region used to measured the flux density of the relic lobe. As shown in Fig. 7, the regions were carefully placed around the cluster to sample a wide range of surface brightness variations. The following average values were obtained for each of the 4 GLEAM sub-band images: -2.41 $\mathrm{Jy},-2.44 \mathrm{Jy},-0.89 \mathrm{Jy}$ and $+1.01 \mathrm{Jy}$, going from the lowest to the highest-frequency subband (since the large-scale Galactic structure is not deconvolved in the GLEAM images, the Galactic contribution can be positive or negative, Hurley-Walker et al. 2017). Finally, these values were used to correct the flux densities measured for the relic lobe in the corresponding sub-band images.

\subsection{Radio spectrum of the relic lobe}



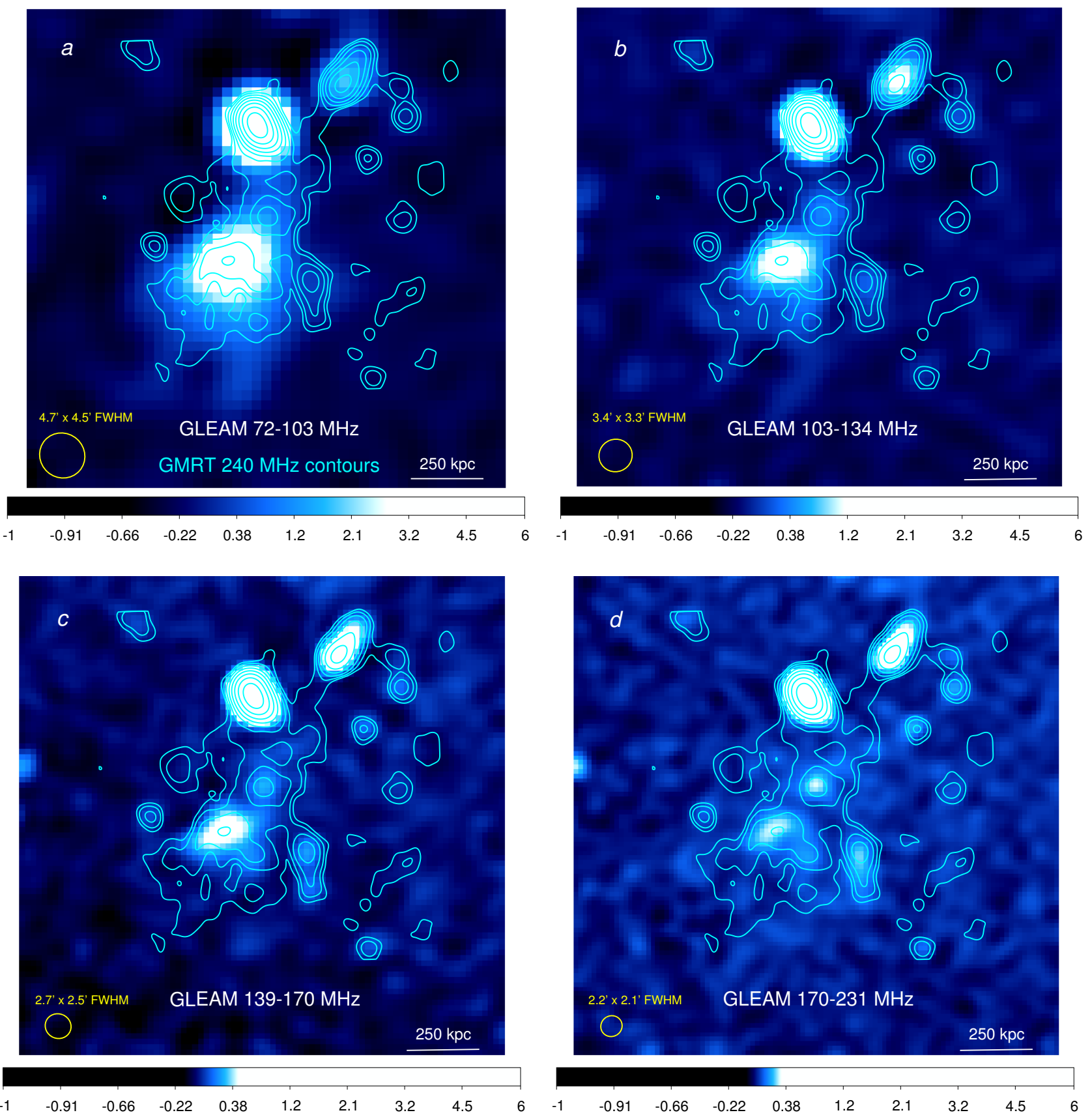

Figure 4. GLEAM images at the central frequencies of (a) $88 \mathrm{MHz}$, (b) $118 \mathrm{MHz}$, (c) $154 \mathrm{MHz}$, (d) 200 $\mathrm{MHz}$ are shown by color; color scale units are Jy. Contours overlay the GMRT $240 \mathrm{MHz}, 2^{\prime}$ resolution image from Fig. $2 b$. The contours are spaced by a factor 2 , starting from $+3 \sigma$. In each panel, the beam size of the GLEAM image is shown by a yellow ellipse. 


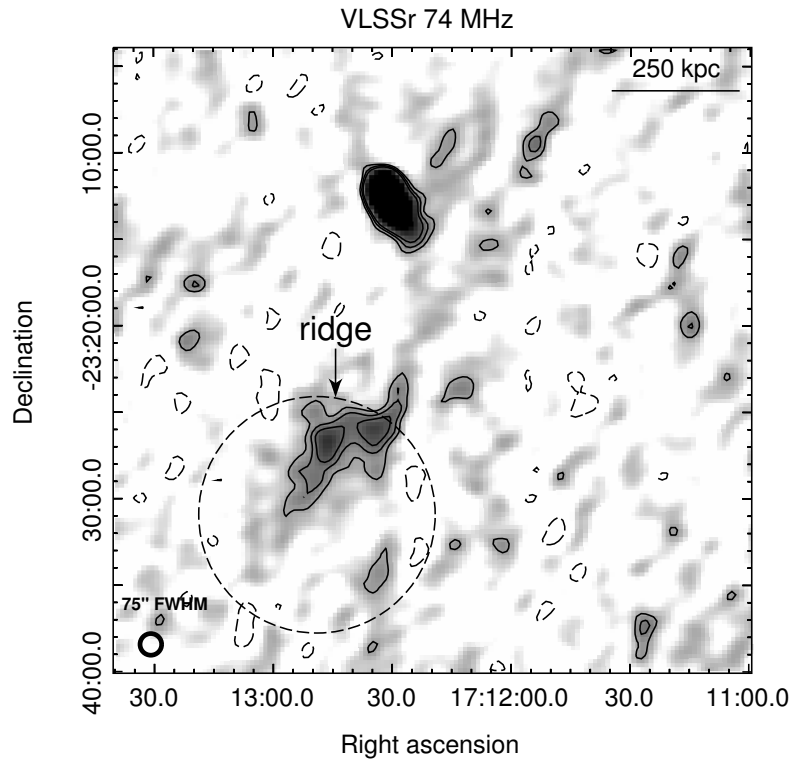

Figure 5. Image at $74 \mathrm{MHz}$ from the VLSSr (gray scale and contours). The restoring beam is $75^{\prime \prime}$ (also shown in black in the bottom-left corner) and the $r m s$ noise $1 \sigma$ level is $180 \mathrm{mJy}^{\text {beam }}{ }^{-1}$. Contours are $-2 \sigma$ (dashed), $+2 \sigma,+3 \sigma$ and $+4 \sigma$. The size of the dashed circle is as in Fig. 2.

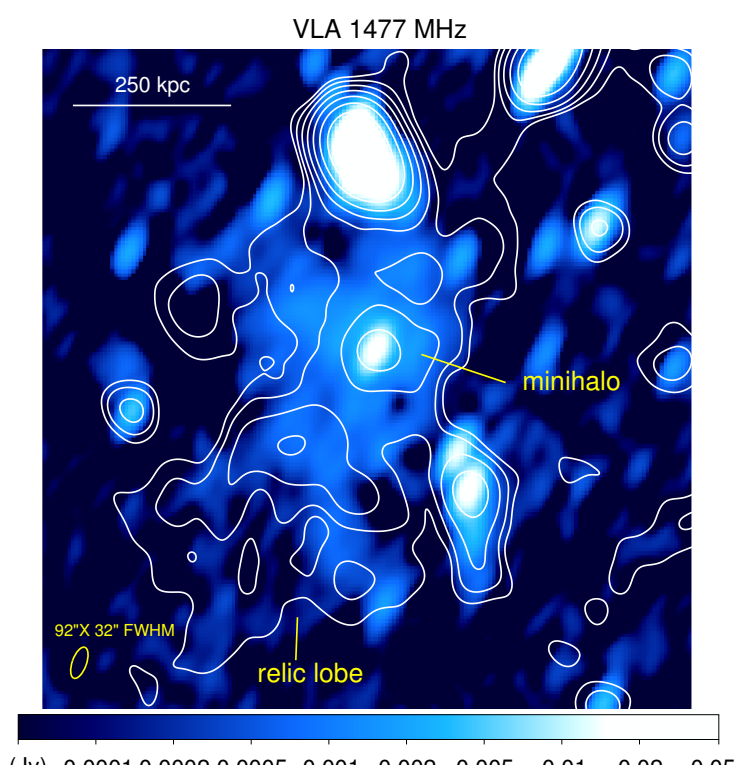

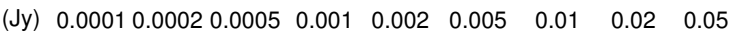

Figure 6. GMRT $240 \mathrm{MHz}$ contour image at $2^{\prime}$ resolution, overlaid on the VLA image at 1477 $\mathrm{MHz}$. The restoring beam of the VLA image is $92^{\prime \prime} \times 32^{\prime \prime}$, in p.a. $-25^{\circ}$ (also shown in yellow) and the $1 \sigma$ noise is $0.1 \mathrm{mJy}^{\text {beam }}{ }^{-1}$.

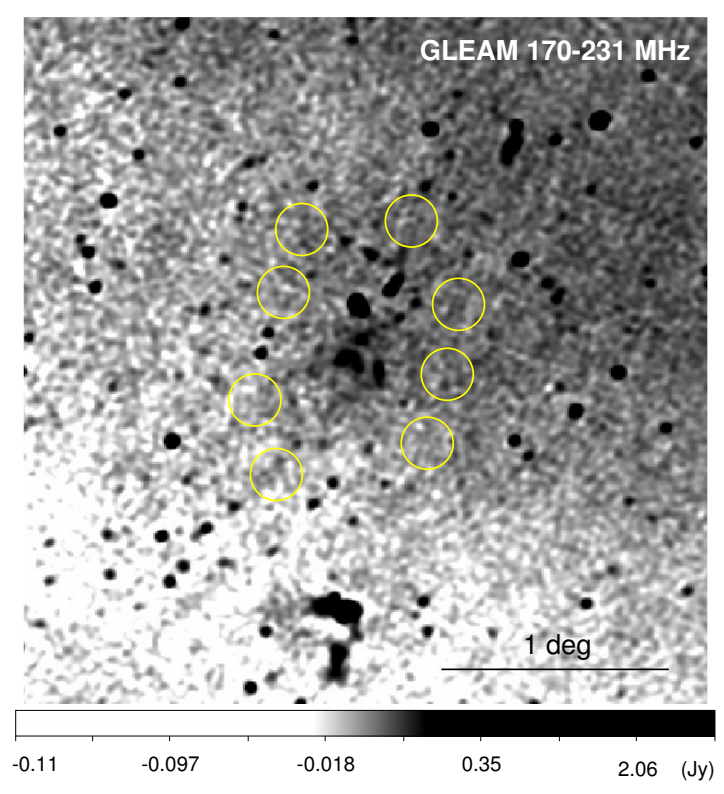

Figure 7. GLEAM $3^{\circ} \times 3^{\circ}$ image in the $170-231$ $\mathrm{MHz}$ sub-band, containing the Ophiuchus cluster. Yellow circles mark the $r=6^{\prime} .8$ regions used to estimate the contribution of the galactic foreground.

Using the flux density measurements in Table 2 , we derived the integrated radio spectrum of the relic lobe between $74 \mathrm{MHz}$ and $1477 \mathrm{MHz}$, shown in Figure 8. The spectrum is very steep and well described by a power law with a spectral index $\alpha_{\text {tot }}=2.4 \pm 0.1$. There is no indication of spectral curvature over the interval of frequency covered by our data. If we use only the low-frequency data points $(74-240 \mathrm{MHz})$, we obtain a slope $\alpha_{\text {low }}=2.7 \pm 0.2$, which is consistent within the errors with $\alpha_{\text {tot }}$. Similarly, if we compute the spectral index from the GLEAM points alone, the resulting slope is $2.5 \pm 0.3$. The two GMRT measurements (blue points) give a flatter spectrum $(\alpha=1.7 \pm 0.4)$. However, the $153 \mathrm{MHz}$ flux is likely underestimated due to the missing structure in the relic lobe at 153 $\mathrm{MHz}$ compared to the $240 \mathrm{MHz}$ image (Figs. 2 and 3). The $153 \mathrm{MHz}$ flux density is also lower than the GLEAM flux density at a similar frequency of $154 \mathrm{MHz}$, though the two values are still consistent within the error bars (Table 2). 
Table 2. Flux density of the relic lobe

\begin{tabular}{lcc}
\hline \hline & $\begin{array}{r}\text { Frequency } \\
(\mathrm{MHz})\end{array}$ & $\begin{array}{c}\text { Flux density } \\
(\mathrm{Jy})\end{array}$ \\
\hline VLSSr & 74 & $18.2 \pm 3.6$ \\
GLEAM & 88 & $11.6 \pm 1.8$ \\
GLEAM & 119 & $5.0 \pm 0.7$ \\
GMRT & 153 & $1.7 \pm 0.3$ \\
GLEAM & 154 & $2.1 \pm 0.5$ \\
GLEAM & 200 & $1.5 \pm 0.3$ \\
GMRT & 240 & $0.78 \pm 0.01$ \\
VLA & 1477 & $0.012 \pm 0.001$ \\
\hline
\end{tabular}

Notes. - At all frequencies, the flux densities were measured within the circular region shown in Fig. 2, with $r=6^{\prime} .8$ and centered on $\mathrm{RA}_{\mathrm{J} 2000}=17 \mathrm{~h} 12 \mathrm{~m} 50.2 \mathrm{~s}$ and $\mathrm{DEC}_{\mathrm{J} 2000}=-23 \mathrm{~d} 31 \mathrm{~m} 13 \mathrm{~s}$. The VLSSr value has been corrected for clean bias and the GLEAM flux densities have been corrected for contamination due to diffuse Galactic synchrotron emission (see text). The flux densities from the GMRT and VLSSr images are on the Scaife \& Heald (2012) scale, the GLEAM fluxes are on the Baars et al. (1977) scale, and the $1477 \mathrm{MHz}$ flux is on the Perley \& Butler (2014) scale. Differences between these scales are estimated to be at most $\sim 5 \%$ (Perley \& Butler 2017).

\subsection{Radio spectrum of the minihalo}

M10 derived the integrated radio spectrum of the central minihalo and measured a possible steepening of the spectral index from $\alpha=$ $1.4 \pm 0.3$ in the $153-240 \mathrm{MHz}$ interval to $\alpha=$ $1.60 \pm 0.05$ in the $240-1477 \mathrm{MHz}$ range. However, the flux density values used by M10 include part of the newly-discovered relic lobe. To investigate how this may impact the behavior of the minihalo spectrum, we rederived the minihalo flux densities after exclusion of the emis-

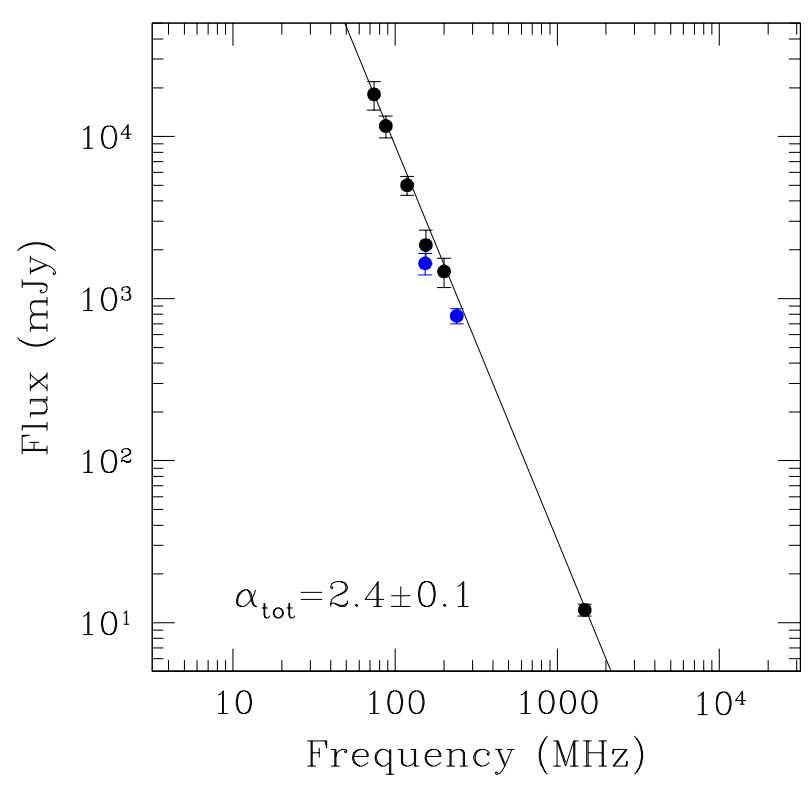

Figure 8. Integrated radio spectrum of the relic lobe between $74 \mathrm{MHz}$ and $1477 \mathrm{MHz}$. The GMRT data points are shown in blue. The solid line is a power law with slope $\alpha_{\text {tot }}=2.4 \pm 0.1$.

sion contributed by the relic lobe. The faint emission associated with the minihalo is detected in both our GMRT images at $153 \mathrm{MHz}$ and $240 \mathrm{MHz}$ (Figs. 1 and 2). The brightest region of the minihalo is also detected in the GLEAM images. However, due to lower angular resolution, it is not possible to separate well the minihalo from the central radio galaxy and bright extended source north of the cluster center. G19 measured a minihalo flux density of $62 \pm 9 \mathrm{mJy}$ at $1477 \mathrm{MHz}$, after subtraction of the contribution from discrete radio sources and relic lobe (see their Appendix A). Following G19, we measure a source- and lobesubtracted flux density of $1224 \pm 203 \mathrm{mJy}$ at $153 \mathrm{MHz}$ and $692 \pm 70 \mathrm{mJy}$ at $240 \mathrm{MHz}$ for the minihalo. The resulting total spectral index is $\alpha_{\text {tot, mh }}=1.32 \pm 0.10$ between $153 \mathrm{MHz}$ and $1477 \mathrm{MHz}$. Contrary to the M10 finding of a possible steepening, the low- and high- 
frequency indices are found to be consistent within the errors $\left(\alpha_{153-240 \mathrm{MHz}}=1.26 \pm 0.43\right.$ and $\left.\alpha_{240-1477 \mathrm{MHz}}=1.33 \pm 0.10\right)$, indicating that the minihalo spectrum can be described by a single power law, at least up to $1477 \mathrm{MHz}$. Highsensitivity observations above this frequency are necessary to better constrain the overall shape of the minihalo spectrum and investigate the presence of a high-frequency steepening, as possibly seen in few other minihalos (e.g., Giacintucci et al. 2014).

\section{X-RAY OBSERVATIONS}

\subsection{Chandra}

The Ophiuchus cluster was observed by Chandra ACIS-I in 2014 for a total exposure of 230 ks (ObsIDs 16142, 16143, 16464, 16626, 16627, 16633, 16634, 16635, 16645). Results from this dataset were first presented by W16. We extracted an image in the $0.5-4 \mathrm{keV}$ band from these data using the standard procedure (e.g., Wang et al. 2016). The image is shown in Figs. 1 and in Fig. 9 with different colors to emphasize the curious concave X-ray brightness edge reported by W16; see also Fig. 1 in W16. This rather sharp edge looks like a boundary of a cavity in the X-ray gas in projection, although the elongation to the south ("southern extension" in Fig. 1) appears to be a separate hydrodynamic disturbance and possibly even a small subcluster, as suggested by W16. We point out in Sect. 5 that the cavity and the hydrodynamic disturbance are not mutually exclusive.

The higher-resolution GMRT $240 \mathrm{MHz}$ contours are overlaid on the Chandra image in Fig. $9 b$, which shows a striking spatial coincidence between the inner edge of the relic lobe (the "ridge") and the X-ray edge that is the boundary of the putative X-ray cavity.

\subsection{XMM-Newton}

The Ophiuchus cluster was observed with XMM-Newton for 37 ks in 2017 (ObsID 0505150101;
Nevalainen et al. 2009). Several offset observations were performed as well, which we do not use here. We extracted images from this dataset using the Extended Source Analysis Software $(X M M \text {-ESAS })^{6}$ package part of SAS version 11.0.0. We used the standard data cleaning and image technique (Snowden et al. 2008). The exposure-corrected and background-subtracted 0.4-7.2 keV image combining the EPIC MOS and pn detectors is shown in Fig. 10. It shows the same concave brightness edge (green arc) and allows us to trace it to somewhat larger scales. The green circle in panel (d) is selected to trace this edge; its radius is $230 \mathrm{kpc}$, which is somewhat bigger than in W16. This difference is not meaningful as it is an extrapolation from a small sector, but our larger radius also happens to encompass the giant low-frequency lobe that is seen in GLEAM (Fig. 10d) and GMRT lower-resolution (Fig. 10c) images.

\subsection{ROSAT}

It is interesting to check the larger-scale structure of the cluster, in order to look for any ghost cavities on the opposite side. The $X M M$ offsets do not cover the outskirts of the Ophiuchus cluster contiguously, so we have checked the archives of other X-ray observatories looking for a better large-scale image. ROSAT PSPC and $A S C A$ have an interesting wide coverage; we extracted a ROSAT image because it has a better angular resolution. We used an archival 0.5-2 keV image from a 4 ks ROSAT PSPC pointing toward the cluster center. It is shown in Fig. 11 with contours from the lowest-band GLEAM image that shows the fossil lobe. The $\mathrm{X}$-ray statistics is clearly not sufficient to attempt a search for a counter-cavity. However, the X-ray image shows that the overall cluster gas distribution is elongated in the direction of

\footnotetext{
${ }^{6}$ http://heasarc.gsfc.nasa.gov/docs/xmm/xmmhp_xmmesas.htm
} 
the lobe, suggesting that the radio and X-ray emission are physically related.

\section{DISCUSSION}

\subsection{Fossil of an enormous AGN outburst?}

The comparison of the radio images and the high-resolution Chandra and XMM X-ray images (Figs. 9,10) shows a large low-frequency radio lobe that fits into the X-ray edge like hand in glove. The spatial coincidence is especially striking between the X-ray edge and the higherresolution GMRT image (Fig. 9b). Neither Chandra nor XMM existing images have the spatial coverage or sensitivity to trace the outer boundaries of the ICM cavity (which would look like a very low-contrast X-ray brightness edge), but the curvature of the visible edge implies that the GLEAM and GMRT radio lobe would fill this putative cavity (Fig. 10) as seen in many clusters with AGN lobes, such as Perseus.

What makes this cavity unique is its size and the energy required to create it. The latter has been estimated by W16 to be $p V \sim 5 \times 10^{61} \mathrm{erg}$, where $p$ comes from the pressure of the ICM around it and $V$ from the Chandra X-ray edge curvature. This value is of course only an orderof-magnitude estimate, as it involves several assumptions, such as where the cavity is located on the line of sight (which should not be too far from the cluster sky plane because it is still visible in the image) and the ICM density and temperature profiles at the relevant $3 \mathrm{D}$ radii. With that caveat in mind, it is interesting to compare the cavity $p V$ and size with the AGN-blown cavities in other clusters. We show such a comparison in Fig. 12, using data for individual cavities (as opposed to their pairs - since we see only one in Ophiuchus) from Rafferty et al. (2006), adding the outer cavities in Hydra A (Wise et al. 2007), the recently-discovered cavities in SPTCLJ0528-5300 (Calzadilla et al. 2019) and a pair of giant cavities in A2204 (Sanders et al. 2009; these are seen in the X-ray but not in the radio, so their nature is still ambiguous). The cavity in Ophiuchus would require an AGN outburst that is an extreme outlier in terms of its total energy - orders of magnitude more energetic than those typically seen in clusters, and 5 times more energetic than the previous record holder, MS $0735+74$.

\subsection{The age of the lobe}

If the lobe has started its life as a buoyant bubble injected near the cluster center by the Ophiuchus central galaxy, it would take it at least $240 \mathrm{Myr}$ to rise to the current radius moving with the sound speed, which is an upper limit on the velocity, so the actual age would be greater than that.

Another method often used to estimate the age of radio galaxy lobes is to look for a break in their radio spectrum and model it. The highestenergy relativistic electrons cool first and the electron power spectrum (and the spectrum of their synchrotron emission) develops an exponential cutoff, which moves to lower energies (and lower radio frequencies) as time passes (e.g., Komissarov and Gubanov 1994). While the radio spectrum of the Ophiuchus cavity is very steep, it is described well by an unbroken power law across our range of frequencies from $74 \mathrm{MHz}$ to $1477 \mathrm{MHz}$, and we do not see any high-frequency cutoff (§3). We cannot simply assume that the cutoff is outside our frequency band, because for an aged source of such an enormous linear size, the total synchrotron spectrum is likely to be the sum of a patchwork of local spectra of different shapes, which could add up to a powerlaw. A large and old radio lobe is likely to contain very nonuniform, filamentary magnetic fields, which would produce different synchrotron spectra and different local synchrotron cooling times even for a single underlying electron power spectrum (e.g., Tregillis, Jones, Ryu 2004). The resulting total radio synchrotron spectrum thus cannot be usefully related to the spectrum of the emit- 

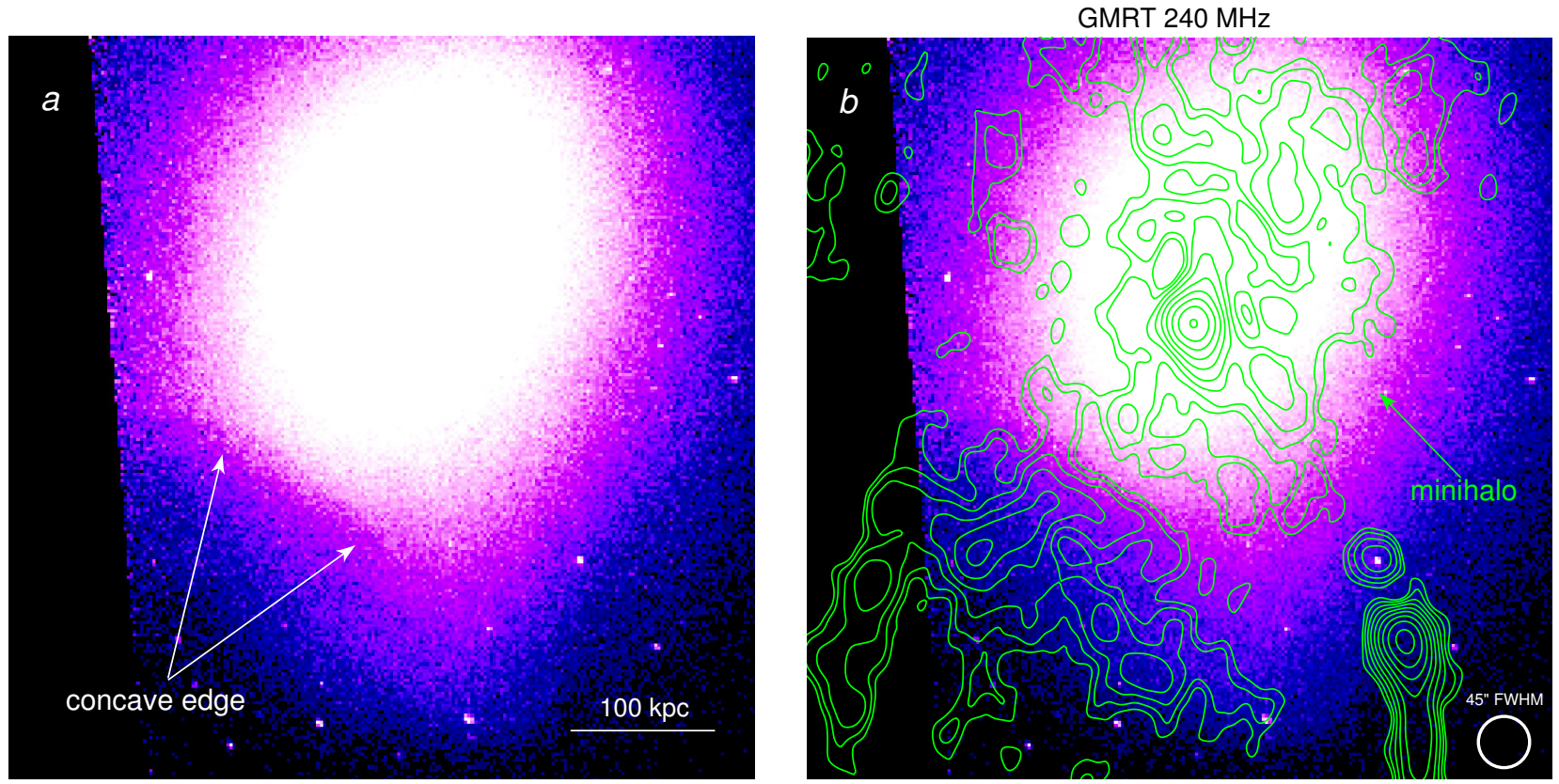

Figure 9. Chandra X-ray image of the Ophiuchus cluster in the $0.5-4 \mathrm{keV}$ band, binned to $4^{\prime \prime}$ pixels. (a) The concave edge, first reported by W16, is shown by arrows. (b) The GMRT $240 \mathrm{MHz}$ image from Fig. $2 a$ is overlaid as green contours. The cluster core contains a radio minihalo. The extended source southeast of the core traces the X-ray edge.

ting electrons (e.g., Eilek \& Arendt 1996; Katz, Stone \& Rudnick 1997).

In this regard, it may be useful to look at a spectral index map with interesting spatial resolution, which would at least remove the spectral averaging over the face of the lobe. We have recently obtained new, deeper and more sensitive observations of this source with the upgraded GMRT (uGMRT) in Band 2 (130-260 $\mathrm{MHz})$ and Band $3(250-500 \mathrm{MHz})$ and we will present the analysis of these new observations in a forthcoming paper.

\subsection{The origin of the cavity}

The spatial coincidence of the X-ray cavity (or at least its inner edge) and the giant, steepspectrum radio lobe is striking and strongly suggests that we have uncovered a fossil of an enormous AGN outburst. However, there are several puzzles in this scenario. First, the central AGN, the supposed culprit, is rather faint and does not show any jets (W16), nor are there any other more powerful AGNs in the vicinity of the relic lobe. However, the central AGN could have been much more active in the past. The X-ray image shows that sloshing has currently displaced the peak of the cluster cool gas away from the $\mathrm{cD}$ galaxy, something that is rarely seen in clusters and that can temporarily starve the AGN of its accretion fuel (Hamer et al. 2012, W16). The gas sloshing seen in the X-ray image may even have been set off by the powerful outburst that has created the giant lobe.

A more difficult puzzle is that we see only one lobe, whereas AGN jets usually come in symmetric pairs and produce a pair of radio lobes in the ICM on two sides of the AGN. Given the likely advanced age of our lobe, we speculate that the counterpart may have propagated into a less-dense ICM on the other side of the cluster and completely faded away. Furthermore, the lobes move together with the ICM in which they are embedded, which breaks the symmetry of the pair (e.g., the outer lobes in $\mathrm{Hy}$ dra A, Nulsen et al. 2005). Strong cluster-wide 

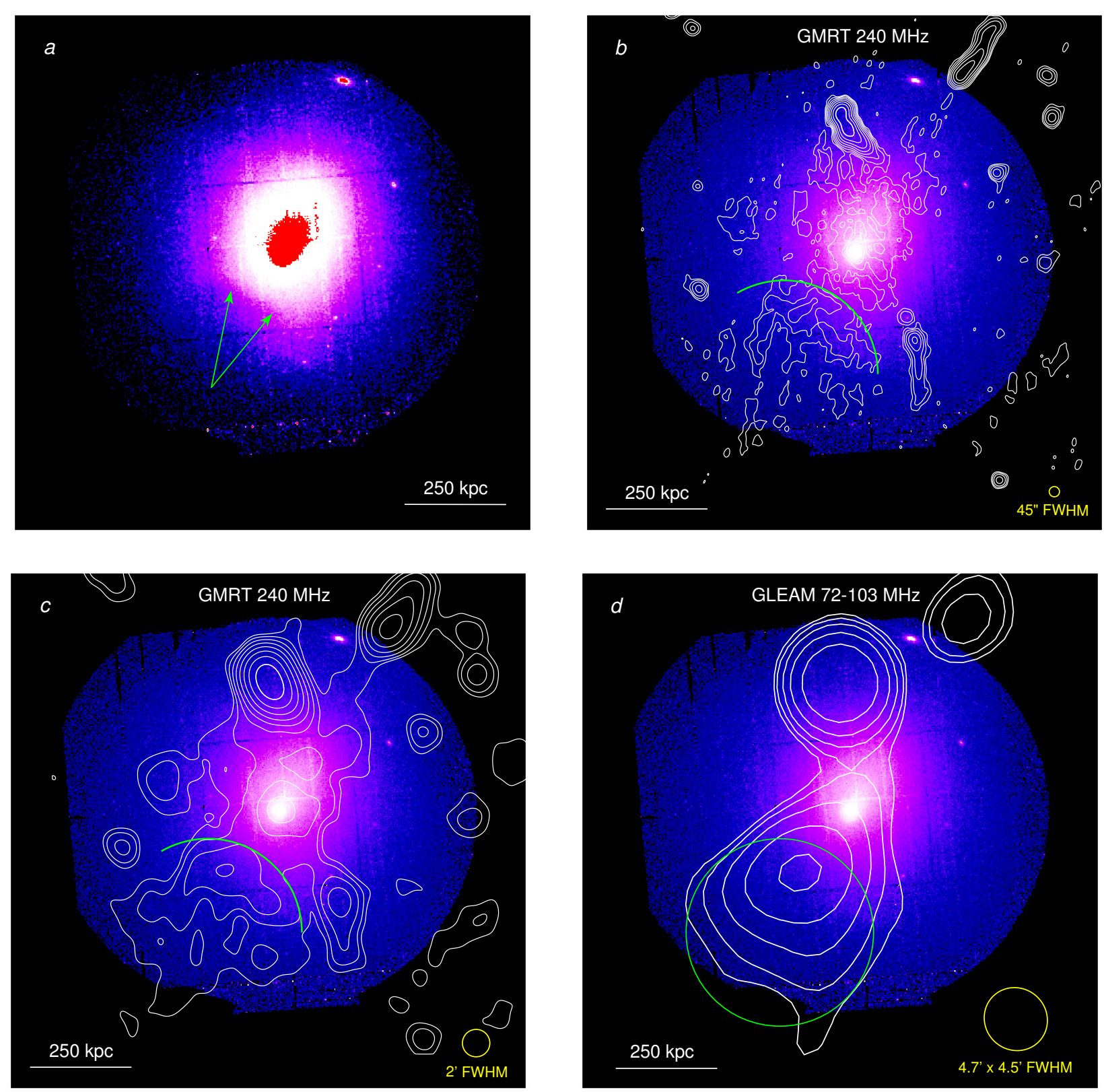

Figure 10. (a) XMM-Newton X-ray image of the Ophiuchus center in the $0.4-7.2 \mathrm{keV}$ band, combining pn and MOS detectors, binned to $5^{\prime \prime}$ pixels. The concave edge is shown with arrows (as in Fig. 9). (b,c,d) The same XMM-Newton image using a different color scale, with the radio contours overlaid from Figs. 2 and $4 a$, spaced by a factor 2 starting from $+3 \sigma$. Radio beams are shown in yellow. The diffuse radio emission southeast of the cool core fills the apparent X-ray cavity and at the lowest frequency becomes a dominant diffuse source. The green circle in (d) encompasses this radio lobe and at the same time traces the X-ray edge in the $X M M$ and Chandra images. The green arcs in (b,c) are a portion of this circle that traces the edge. 


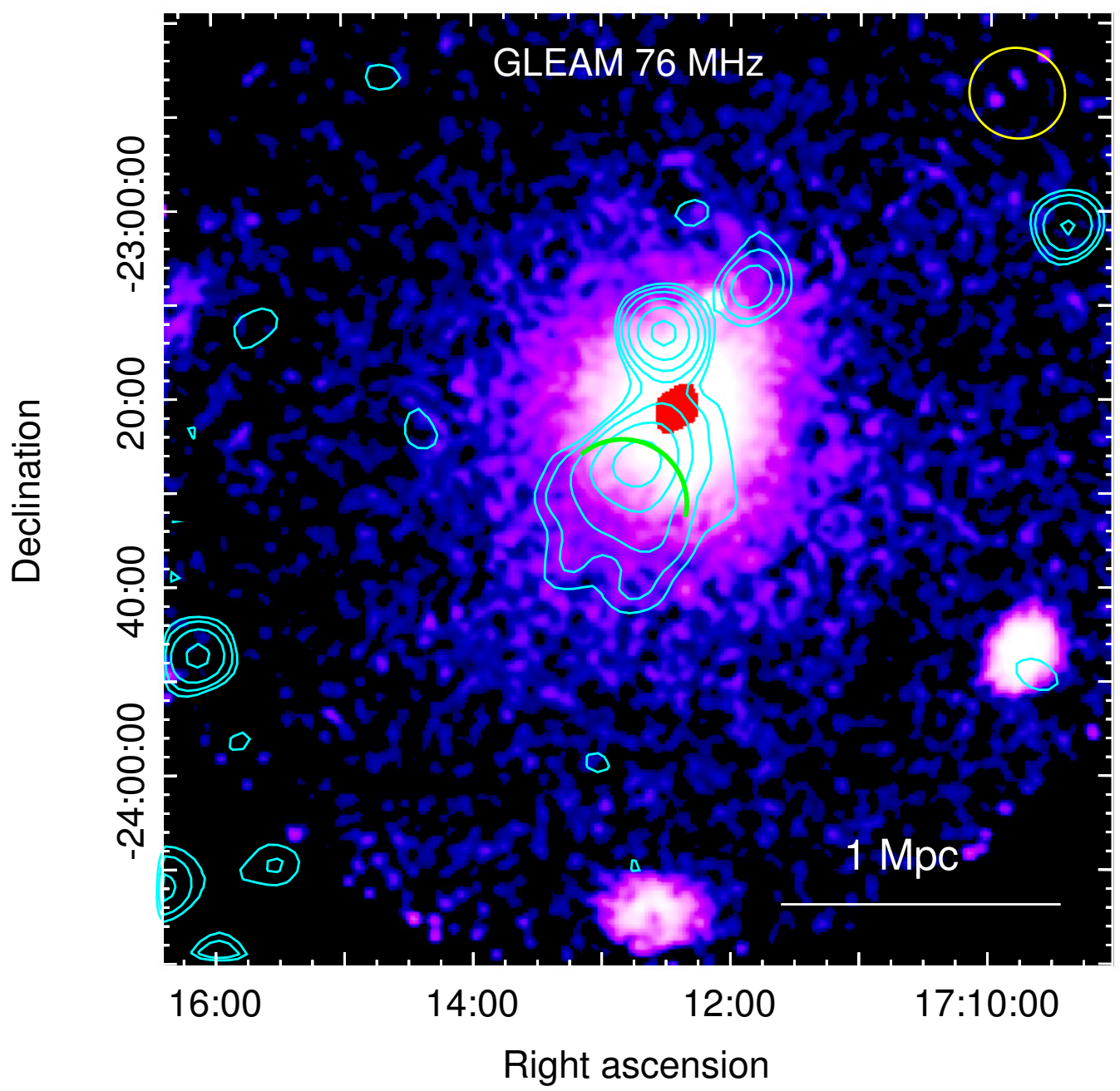

Figure 11. MWA GLEAM contours in the $72-80 \mathrm{MHz}$ band overlaid on the ROSAT PSPC X-ray image that shows the cluster structure on larger scales. The cluster shows elongation on the side of the radio lobe. The green arc traces the X-ray edge seen in the Chandra and XMM images. (The two X-ray blobs at bottom and lower-right are point sources affected by the degraded angular resolution at the edges of the ROSAT field of view.) Radio contours are spaced by a factor of 2 starting from 400 mJy beam ${ }^{-1}$. The radio beam (yellow ellipse) is $5^{\prime} .1 \times 4^{\prime} .8$, in p.a. $15^{\circ}$. 

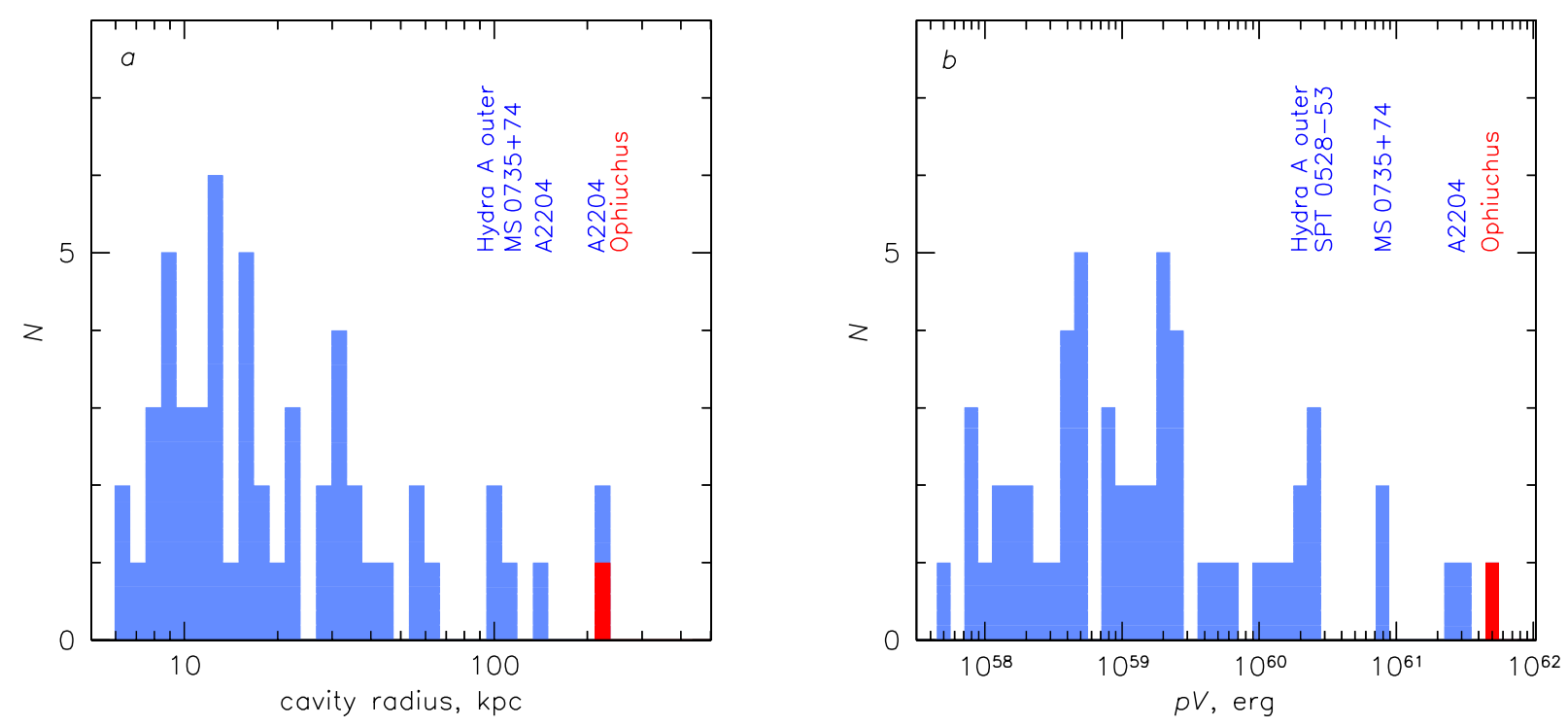

Figure 12. The distribution of known AGN cavities in galaxy clusters: (a) by their size and (b) by their energy ( $p V$ for each cavity). The biggest cavities are labeled. Data from Rafferty et al. (2006) supplemented by the Hydra A outer cavities from Wise et al. (2007), the recently-discovered cavities in SPT-CLJ05285300 from Calzadilla et al. (2019) and the possible ghost cavities in A 2204 from Sanders et al. (2009). The Ophiuchus cavity $p V$ estimate is from W16, while the size is from this work (we find a somewhat larger size than W16, thus the $p V$ value may be an underestimate). The X-ray based cavity sizes are uncertain and the $p V$ estimates even more so, but the Ophiuchus cavity is certainly an enormous outlier.

bulk gas motions, such as those generated by a merger, may have moved the counterpart lobe somewhere in the outskirts, where it faded. An exotic alternative is that the radio galaxy that gave rise to this bubble is not the $\mathrm{cD}$ galaxy, but some other galaxy perhaps in the cluster outskirts; the counterpart lobe would then fade quickly in the low-density gas far from the cluster center.

Another serious problem was posed by W16 - how did the cool core survive such an energetic event? There are clusters whose cores appear to have been torn to pieces by AGN outbursts (e.g., A2390, Vikhlinin et al. 2005). The Ophiuchus cool core has a steeper radial density and temperature gradient than most cool cores (W16), which may make it more stable against a disruption that comes from the side rather than from the nucleus. One can imagine long jets from the central AGN piercing the cool core and depositing energy and blowing bubbles right outside the core. On smaller linear scales, this is observed in some cluster galaxies with dense gaseous coronae (e.g., Sun et al. 2007, Sanders et al. 2014) or in remnant compact cool cores (e.g., Cheung et al. 2019), which are pierced by jets emitted by the AGN without disrupting the corona/compact core, and creating bubbles right outside.

The Ophiuchus core does exhibit stronger sloshing motions than most cool cores. It may have been triggered by an asymmetric pair of expanding bubbles deposited outside the core, which may have given the core a push from the side while avoiding destroying the core. It would be interesting to explore such a possibility using hydrodynamic simulations.

On larger linear scales, the ROSAT image (§4.3) exhibits a curious elongation in the exact direction of the giant lobe. There are two 
interesting possibilities here. One is the analogy with Hydra A, where a pair of giant radio bubbles is ensconced in an X-ray cocoon (see Fig. 2 in Nulsen et al. 2005), whose outer edge is a weak shock front (Simionescu et al. 2009). Another possibility is cluster-wide sloshing, such as recently found in Perseus (Simionescu et al. 2012) and A2142 (Rossetti et al. 2013), both of which exhibit cold fronts $\sim 1 \mathrm{Mpc}$ from the center. If the enormous AGN outburst managed to move the cool core and make it slosh, it might induce cluster-wide sloshing as well its mechanical energy approaches that of minor mergers.

We note here that the hydrodynamic instability of a sloshing front, proposed by W16 to explain the concave brightness edge, and our radio bubble explanation, are not mutually exclusive. The "subcluster," or the surface brightness excess south of the core at one end of the concave feature (Fig. 1b in W16 and "southern extension" in our Fig. 1), exhibits a morphology that makes it likely to be a splash of cool gas from the core generated by the expansion of the bubble and the resulting major hydrodynamic disturbance of the cluster.

\section{CONCLUSIONS}

The "gentle" AGN feedback is the current preferred solution for the cluster cooling flow problem. In this scenario, the mechanical energy of multiple outbursts from the central AGN provides gentle, steady, self-regulated heating of the cool gas in the core that exactly compensates for fast X-ray radiative cooling of that gas (e.g., McNamara \& Nulsen 2012; Fabian 2012, e.g.; Voit et al. 2015; Gaspari et al. 2017). Ophiuchus offers a dramatic example that is far outside this scenario. We found evidence of an extraordinarily powerful past AGN outburst that has deposited its energy outside the cool core, without completely disrupting the core.
The evidence comes in the form of a giant radio lobe with a very steep spectrum, discovered by MWA and confirmed by GMRT, which coincides spatially with an apparent cavity in the cluster gas.

This enormous fossil radio source may be an early example of a new class of sources to be uncovered by the ongoing highly sensitive lowfrequency surveys of galaxy clusters. Their counterpart cavities in the X-ray gas outside the cluster bright central regions will have very low brightness contrast. To detect them in the $\mathrm{X}$-ray, it would require a combination of high angular resolution and large collecting area to obtain the sufficient photon statistics. For example, Athena's imaging instrument (Barcons et al. 2017) and the AXIS concept (Mushotzky et al. 2019) will be able to detect such features.

\section{Acknowledgements.}

We thank the anonymous referee. This scientific work makes use of the Murchison Radioastronomy Observatory, operated by CSIRO. We acknowledge the Wajarri Yamatji people as the traditional owners of the observatory site. Support for the operation of the MWA is provided by the Australian Government (NCRIS), under a contract to Curtin University administered by Astronomy Australia Limited. We acknowledge the Pawsey Supercomputing Centre which is supported by the Western Australian and Australian Governments. GMRT is run by the National Centre for Astrophysics of the Tata Institute of Fundamental Research. The National Radio Astronomy Observatory is a facility of the National Science Foundation operated under cooperative agreement by Associated Universities, Inc. Basic research in radio astronomy at the Naval Research Laboratory is supported by 6.1 Base funding. Q. H. S. Wang acknowledges the support of NASA via Chandra grant AR5-16013X. 


\section{REFERENCES}

Ascasibar, Y., \& Markevitch, M. 2006, ApJ, 650, 102

Baars, J. W. M., Genzel, R., Pauliny-Toth, I. I. K., et al. 1977, A\&A, 500, 135

Barcons, X., Barret, D., Decourchelle, A., et al. 2017, AN, 338, 153

Briggs, D. S. 1995, Bulletin of the American Astronomical Society, 27, 112.02

Calzadilla, M. S., McDonald, M., Bayliss, M., et al. 2019, ApJL, 887, L17

Chandra, P., Ray, A., \& Bhatnagar, S. 2004, ApJL, 604, L97

Cheung, C. C., Giacintucci, S., \& Clarke, T. E. 2019, ApJ, 875, 108

Eckert, D., Produit, N., Paltani, S., et al. 2008, A\&A, 479, 27

Eilek, J. A., \& Arendt, P. N. 1996, ApJ, 457, 150

Fabian, A. C., Sanders, J. S., Allen, S. W., et al. 2003, MNRAS, 344, L43

Fabian, A. C. 2012, ARA\&A, 50, 455

Fujita, Y., Hayashida, K., Nagai, M., et al. 2008, PASJ, 60, 1133

Gaspari, M., Temi, P., \& Brighenti, F. 2017, MNRAS, 466, 677

Giacintucci, S., Markevitch, M., Brunetti, G., et al. 2014, ApJ, 795, 73

Giacintucci, S., Markevitch, M., Cassano, R., et al. 2017, ApJ, 841, 71

Giacintucci, S., Markevitch, M., Cassano, R., et al. 2019, ApJ, 880, 70 (G19)

Govoni, F., Murgia, M., Markevitch, M., et al. 2009, A\&A, 499, 371 (G09)

Hamer, S. L., Edge, A. C., Swinbank, A. M., et al. 2012, MNRAS, 421, 3409

Hurley-Walker, N., Callingham, J. R., Hancock, P. J., et al. 2017, MNRAS, 464, 1146

Katz-Stone, D. M., \& Rudnick, L. 1997, ApJ, 488, 146

Komissarov, S. S., \& Gubanov, A. G. 1994, A\&A, 285,27

Lane, W. M., Cotton, W. D., van Velzen, S., et al. 2014, MNRAS, 440, 327

McNamara, B. R., Nulsen, P. E. J., Wise, M. W., et al. 2005, Nature, 433, 45

McNamara, B. R., \& Nulsen, P. E. J. 2012, New Journal of Physics, 14, 055023

Million, E. T., Allen, S. W., Werner, N., \& Taylor, G. B. 2010, MNRAS, 405, 1624
Murgia, M., Eckert, D., Govoni, F., et al. 2010, A\&A, 514, A76 (M10)

Murgia, M., Parma, P., Mack, K.-H., et al. 2011, A\&A, 526, A148

Mushotzky, R. F., Aird, J., Barger, A. J., et al. 2019, arXiv e-prints, arXiv:1903.04083

Nevalainen, J., Eckert, D., Kaastra, J., Bonamente, M., \& Kettula, K. 2009, A\&A, 508, 1161

Nulsen, P. E. J., McNamara, B. R., Wise, M. W., \& David, L. P. 2005, ApJ, 628, 629

Pérez-Torres, M. A., Zandanel, F., Guerrero, M. A., et al. 2009, MNRAS, 396, 2237 (P09)

Perley, R., \& Butler, B. 2014, Astronomical Society of India Conference Series, 361

Perley, R. A., \& Butler, B. J. 2017, ApJS, 230, 7

Rafferty, D. A., McNamara, B. R., Nulsen, P. E. J., et al. 2006, ApJ, 652, 216

Rossetti, M., Eckert, D., De Grandi, S., et al. 2013, A\&A, 556, A44

Sanders, J. S., Fabian, A. C., \& Taylor, G. B. 2009, MNRAS, 393, 71

Sanders, J. S., Fabian, A. C., Sun, M., et al. 2014, MNRAS, 439, 1182

Scaife, A. M. M., \& Heald, G. H. 2012, MNRAS, 423, L30

Simionescu, A., Roediger, E., Nulsen, P. E. J., et al. 2009, A\&A, 495, 721

Simionescu, A., Werner, N., Urban, O., et al. 2012, ApJ, 757, 182

Snowden, S. L., Mushotzky, R. F., Kuntz, K. D., et al. 2008, A\&A, 478, 615

Su, H., Hurley-Walker, N., Jackson, C. A., et al. 2017, MNRAS, 465, 3163

Sun, M., Jones, C., Forman, W., et al. 2007, ApJ, 657, 197

Tregillis, I. L., Jones, T. W., \& Ryu, D. 2004, ApJ, 601, 778

Vikhlinin, A., Markevitch, M., Murray, S. S., et al. 2005, ApJ, 628, 655

Voit, G. M., Donahue, M., Bryan, G. L., et al. 2015, Nature, 519, 203

Walker, S. A., Hlavacek-Larrondo, J., Gendron-Marsolais, M., et al. 2017, MNRAS, 468, 2506

Wang, Q. H. S., Markevitch, M., \& Giacintucci, S. 2016, ApJ, 833, 99 
Wayth, R. B., Lenc, E., Bell, M. E., et al. 2015, PASA, 32, e025

Werner, N., Zhuravleva, I., Canning, R. E. A., et al. 2016, MNRAS, 460, 2752 (W16)
Wise, M. W., McNamara, B. R., Nulsen, P. E. J., et al. 2007, ApJ, 659, 1153

ZuHone, J. A., Markevitch, M., \& Johnson, R. E. 2010, ApJ, 717, 908 